\title{
Infraestrutura de biossegurança para agentes biológicos em hospitais do sul do Estado da Bahia, Brasil
}

\author{
Biosecurity infrastructure for biological agents in hospitals from the south of Bahia State, Brazil \\ Infraesctructura de bioseguridad para los agentes biológicos en hospitales del sur del Estado de Bahia, Brasil
}

\author{
Maridalva de Souza Penteado', Tânia Cristina Oliveira" \\ 'Universidade Estadual de Santa Cruz. Departamento de Ciências da Saúde. Ihéus, BA \\ "Universidade de Taubaté. Departamento de Medicina. Taubaté, SP
}

Submissão: 05/05/2008

Aprovação: 09/01/2010

\section{RESUMO}

O objetivo do estudo foi descrever a infraestrutura de biossegurança em hospitais localizados na Região Sul do Estado da Bahia, Brasil. Tratou-se de um estudo descritivo-exploratório realizado em comissões de controle de infecções hospitalares e comissão institucional de prevenção a acidentes Quanto à existência de normas escritas, realização de treinamentos para essas mesmas preconizações, existência de registros institucionais concernentes à biossegurança e existência de práticas de atenção à saúde dos profissionais e isolamentos de pacientes. Os hospitais são comparados segundo a presença de cada um dos itens analisados de acordo com sua classificação por porte, vinculação jurídico-financeira, dentre outras. Conclui-se Que a situação geral é precária Quanto à presença dos itens investigados, o que impele a considerar a necessidade de que se leve em conta, numa análise de Qualidade hospitalar, também o cuidado com a vida dos seus trabalhadores.

Descritores: Exposição a agentes biológicos; Infraestrutura; Controle de risco.

\section{ABSTRACT}

The purpose of the study was to describe the biosecurity infrastructure in hospitals located in the South Region of Bahia State, Brazil. That was a descriptive-exploratory study carried out in commissions hospital infections control and institutional prevention commission of accidents about the existence of written norms, the accomplishment of training, the existence of concernments institutional registers to the biosecurity, and the existence of practical attention to the health of the professionals and isolated patients. Hospitals are compared according to the presence of each one of the itens under analysis as its classification, legal-financial issues, etc. It was conclude that the general situation is precarious regarding the presence of the items investigated, that impels to consider the necessity that must take into account an analysis of hospital Quality, and also the care of the life of its workers.

Key words: Exposure to biological agents; Infrastructure; Risk management.

\section{RESUMEN}

El objetivo del estudio fue describir la infraestructura de bioseguridad en hospitales de la Región Sur del Estado de Bahia, Brasil. Fue realizada una investigación descriptiva-exploratória en las comisiones de control de infecciones hospitalarios y en las comisiónes institucionales de prevención de accidentes sobre la existencia de normas escritas, la realización de entrenamientos para esas mismas preconizaciones, la existencia de registros institucionales concernientes a la bioseguridad, la existencia de practicas de atención a la salud de los profesionales y apartamiento de pacientes. Los hospitales son son comparados según la presencia de cada uno de los itens analizados en conformidad con su porte, vinculación jurídico financiera, entre otras. Se concluye que la situación general es precaria cuanto a la presencia de los itens investigados, lo Que lleva a considerar la necesidad de que se lleve en cuenta, la análisis de la calidad hospitalario, también el cuidado con la vida de sus trabajadores.

Descriptores: Exposición a agentes biológicos; Infraestructura; Control de riesgo. 


\section{INTRODUÇÃO}

Em Que pesem a ausência de um modelo de avaliação Que leve em conta as características hospitalares enQuanto meio seguro de atuação profissional frente à problemática dos riscos biológicos e, ainda, a conhecida variação Qualitativa das instituições hospitalares nacionais e as suas demandas específicas, propõe-se, neste estudo, a investigar a dimensão do problema biossegurança em hospitais. A biossegurança é entendida como o conjunto de normas e procedimentos considerados seguros e adequados à manutenção da saúde, em atividades de risco de aquisição de doenças profissionais ${ }^{(1)}$. Antes de uma disciplina em sentido estrito, um campo de realização de práticas técnicas e sociais destinadas a conhecer e controlar os riscos que o trabalho em saúde pode aportar ao meio e à vida. Práticas essas Que dependem de uma série de fatores Que incluem elementos conjunturais e estruturais.

Levando-se em conta Que se vislumbra, em última instância, a melhoria da Qualidade da assistência prestada, não há como se pensar em melhorar a Qualidade do atendimento, sem melhorar a Qualidade do trabalho em saúde no seu mais amplo sentido. O fornecimento de cuidados de saúde satisfatórios, de maneira desejável tem obrigatória intersecção com a adoção de medidas de biossegurança, porQue se assim não for, o cuidado poderá ser prestado sob uma plêiade de riscos para o profissional e, em subsequência, também para o próprio usuário do serviço de saúde.Por outro lado, as preocupações emergentes com a biossegurança, e sua inclusão na visão geral da Qualidade dos serviços não se constituem em um significado restrito. Fazem parte de uma tendência mundial, sustentada por uma mudança de paradigma cultural, pelo Que se vem advogando e promovendo novos padrões de comportamento, diante das Questões da preservação do meio ambiente, da própria vida e das relações externas à comunidade ${ }^{(1)}$. Observe-se Que os campos compreendidos pela biossegurança e pela gestão de Qualidade estão unidos um ao outro, desde sua origem através do conceito de boas práticas. As boas práticas dependem de fatores sociais (construção, por exemplo, de formas de comportamento), fatores pessoais (Que incluem valores individuais atribuídos às práticas pelo sujeito) e fatores ambientais (estrutura de apoio e tecnologia mínima disponível).

Importante é dar relevância às diversas evidências de Que os profissionais atuantes na área da saúde e, particularmente em hospitais, estão sujeitos a adeuirir infecções,em particular as hepatites B e C, a AIDS e a tuberculose, especialmente. As primeiras pelas incidências que podem ser consideradas altas e subnotificadas; a AIDS ainda pelo espectro de doença fatal e a tuberculose, por suas diversas consequências sobre a vida dos profissionais.

\section{OBJETIVO}

Descrever a infraestrutura de biossegurança em hospitais localizados na Região Sul do Estado da Bahia, Brasil.

\section{METODOLOGIA}

A pesquisa constou de estudo descritivo-exploratório empreendido nos hospitais localizados na área de abrangência da Sétima
Diretoria Regional de Saúde da Bahia (7 $7^{\mathrm{a}}$ DIRES). Para tal utilizouse como unidades elementares de análise, os hospitais relacionados à citada diretoria, responsável pela supervisão das ações de saúde de vinte e dois municípios, localizando-se sua sede regional na cidade de Itabuna. A rede hospitalar em pauta constitui-se de vinte e sete instituições, nove delas localizadas no município pólo (Itabuna) e as demais distribuídas uma para cada um dos demais municípios da circunscrição. Foram visitadas, para fins do estudo, vinte e cinco das vinte e sete instituições existentes.

Para a análise das estruturas hospitalares relacionadas à biossegurança, foi utilizado formulário adaptado ${ }^{(2-3)}$, o qual constou, além de dados para categorização dos hospitais, de Questões referentes à estrutura de biossegurança. $\mathrm{O}$ formulário foi aplicado aos enfermeiros dos respectivos grupos executores de controle de infecção, Quando existente, na falta deste ao presidente da $\mathrm{CCIH} \mathrm{e,}$ finalmente, não existindo $\mathrm{CCIH}$, ao coordenador de enfermagem.

Antecedeu a pesquisa sua aprovação pelo comitê de ética e pesquisa da Faculdade de Saúde Pública da Universidade de São Paulo, haja vista de tratar-se de trabalho a ela vinculado. Obtevese ainda, antes da coleta de dados, autorização das administrações institucionais envolvidas, para Que a coleta de dados fosse procedida, bem como aquiescência pessoal, por parte dos respondentes, de termo de consentimento livre e esclarecido.

Para o tratamento dos dados, procedeu-se além de análise da proporção dos itens considerados para fins do estudo, presentes nos hospitais, à análise das distinções e similaridades entre hospitais. Essa, de acordo com as subdivisões segundo porte, vinculação administrativo-financeira, ou seja, se públicos, privados e filantrópicos, natureza geral ou especializada, e, ainda, segundo o predomínio da clientela atendida sob o ponto de vista da remuneração pelos serviços, do corpo clínico do hospital (se aberto e fechado), de sua caracterização enQuanto se prestar ou não a campo formal de ensino de profissionais da área de saúde e, por fim, do ponto de vista da sua localização geográfica, se na cidade considerada como pólo regional, Itabuna, ou fora dela. Complementarmente também analisamos os hospitais segundo subdivisão com ou sem $\mathrm{CCIH}$ e grupo executor de controle de infecção hospitalar.

Para a análise dos efeitos das características hospitalares sobre a ocorrência de itens concernentes à biossegurança, os hospitais, uma vez agrupados, foram, a princípio, analisados em relação a cada Questão em particular. Para tal, utilizamos o teste QuiQuadrado ou exato de Fisher. Ao invés de fixar o nível de significância para a rejeição da hipótese estatística, optou-se por colocar no texto o nível de significância da amostra (p valor) $)^{(4)}$.

\section{RESULTADOS}

\section{Infraestrutura Organizacional - Caracterização das $\mathrm{CClHs}$ e das CIPAs}

Dos vinte e cinco hospitais estudados, dezenove (76\%) possuiam CCIH e seis (24\%) não dispunham do órgão. Todos os hospitais privados $(n=7)$ tinham formalmente constituída $\mathrm{CCIH}$, ao passo Que nos hospitais públicos $(n=12)$ e filantrópicos $(n=6)$, elas estavam presentes em $66,7 \%$ das instituições. Há também variação na existência de $\mathrm{CCIH}$ nos hospitais Quando distribuídos por tamanho: os dois únicos hospitais de grande porte (100,0\%) tinham 
$\mathrm{CCIH}$, seguidos pelos de tamanho médio $(n=9), 80 \%$ e daqueles de pequeno porte $(n=14) 72,4 \%$.

Apenas três $(15,8 \%)$, das dezenove CCIHs, tinham papéis delimitados entre os seus participantes, nas demais, o enfermeiro assumia as funções inerentes à Comissão.

As atividades dos enfermeiros nas comissões de controle de infecções hospitalares demonstram uma concentração nas ações da vigilância epidemiológica das Ihs, bem como na elaboração de planilhas destinadas à prestação de informações aos órgãos de direito, a despeito de demais ações como a educação continuada (somente três CCIHs-15,8\%, a referiram) e implementação de isolamentos (apenas uma CCIH confirmou atuação nessa função). Detectamos ainda Que nove desses mesmos enfermeiros, realizaram treinamento/curso em temas afetos à biossegurança, sendo Que os profissionais foram treinados em maior proporção nos hospitais privados $(57,1 \%$ de um total de sete $\mathrm{CCIHs})$, e nos hospitais filantrópicos (50,0\% de um total de Quatro $\mathrm{CCIHs})$, a despeito dos hospitais públicos, cuja percentagem de enfermeiros das respectivas CCIHs treinados foi de $37,5 \%$ de um total de oito. Apenas três, das vinte e cinco instituições (12,0\%), uma de médio porte (filantrópica) e duas de grande porte (uma pública e uma filantrópica), possuiam grupo executor de controle de infecção hospitalar. As composições desses mesmos grupos restringiam-se basicamente ao enfermeiro atuando em dedicação integral (36 horas semanais) e um médico em dedicação parcial para cada um dos hospitais filantrópicos.

Nenhuma das vinte e cinco instituições estudadas possuía serviço médico ocupacional ou assemelhado e somente três hospitais (12\%) eram dotados de Comissão Interna de Prevenção de Acidentes (CIPA) e em uma dessas CIPAS pelo menos um de seus membros realizou curso/treinamento em biossegurança.

Presença de normas, treinamentos, registros, rotinas e infraestrutura física relativas à biossegurança

No Que se refere aos demais itens aqui considerados para a configuração de uma adequada estrutura de biossegurança, observamos baixos percentuais afirmativos para a existência de normalizações escritas relativas à biossegurança, consideradas para fins do estudo nas instituições (Apêndice). Chama a atenção o fato de vinte e dois hospitais $(88,0 \%)$ não disporem sequer das normas concernentes às precauções padrão. Resultado ainda mais modesto ocorreu com as normas relativas às precauções baseadas na transmissão, ausentes em vinte e três nosocômios $(92,0 \%)$. As normas referentes às condutas frente aos casos de acidentes de profissionais com materiais pérfuro-cortantes, embora presentes em número maior de instituições que as demais, tinham, também, tímida presença nos serviços hospitalares (oito instituições -32,0\%). Procedido tratamento estatístico, verificamos diferenças significativas ao nível de $7 \%$ de probabilidade Quando estudadas a presença de normas escritas, em seu conjunto, Quando os hospitais são categorizados por porte, com melhores resultados os hospitais grandes, seguidos pelos de médio e pequeno porte e diferenças ao nível de 2,0\% $(p=0,02)$ entre os hospitais categorizados por vinculação financeira, com melhores performances para as instituições filantrópicas, seguidas pelos hospitais privados e, por último, os públicos. Agrupados pela característica de se constituírem ou não em campo de práticas para atividades de cursos na área da saúde ( $n=3$ e 22 respectivamente), os hospitais demonstraram-se com diferenças significativas em nível de $0,4 \%(p=0,004)$ para o conjunto de normas escritas, com melhores resultados para o primeiro grupo. Verificamos, igualmente, diferença estatisticamente significativa ao nível de $6,0 \%(p=0,06)$ para o conjunto de Questões Quando esses são separados por localização geográfica, considerada como situados na cidade pólo, Itabuna, ou fora dela. As instituições aí localizadas apresentaram desempenho melhor do que aquelas localizadas nos demais municípios.

Ao se analisar a presença de normas escritas nas instituições com e sem $\mathrm{CCIH}$, não se detectam diferenças estatisticamente significativas. Separados os hospitais de acordo com a existência de grupo executor de controle de IH detectamos, contudo, diferenças, com significância estatística ao nível de $1,0 \%(p=0,01)$, com melhor desempenho para os hospitais providos dos respectivos serviços. Características outras dos hospitais, como ser geral ou especializado ( $n=22$ e 3 , respectivamente), ter corpo clínico aberto ou fechado ( $n=3$ e 22 respectivamente), clientela atendida $(n=16)$ para atendimentos exclusivos ao SUS; uma somente para pacientes particulares e convênios privados e oito para todas as fontes de pagamento), não separam os hospitais de forma estatisticamente significativa para o item aQui apresentado.

Por outro lado, Quando se avalia a realização de treinamento de pessoal para as precauções padrão, verificamos, muito embora com percentuais também modestos, referência afirmativa em percentual de $40 \%$ dos hospitais. O mesmo fenômeno é observado para as precauções baseadas na transmissão, cujo percentual de realização de treinamento chega a $36,0 \%$. O preparo de pessoal para triagem precoce de pacientes suspeitos de tuberculose, em contrapartida, não era praticado em nenhum dos hospitais estudados.

Não se demonstram, ainda, diferenças estatisticamente significantes dentre os grupos de hospitais para as Questões afetas ao treinamento como um todo, bem como para os subitens considerados individualmente (precauções padrão, precauções baseadas na transmissão e triagem precoce de pacientes suspeitos de tuberculose), ainda Que as médias apontem uma ligeira tendência de resultados pouco mais promissores, pelo menos para as precauções padrão, nos hospitais de grande e médio porte. $\mathrm{O}$ mesmo ocorre ao se analisar o conjunto das Questões atinentes à realização de treinamentos, tomados os hospitais categorizados por vinculação administrativa financeira, embora haja tendência de melhores resultados para os hospitais filantrópicos.

Não se diferenciam os hospitais com e sem $\mathrm{CCIH}$, para o item treinamento no seu conjunto, ainda Que os percentuais apontem para uma ligeira tendência de melhores resultados em favor do primeiro grupo. As instituições agrupadas segundo a existência ou não de grupo executor de controle de infecção hospitalar, em contrapartida, diferenciam-se no nível de $6,0 \%(p=0,06)$ de significância estatística, com melhor desempenho para os primeiros Quando analisados os subitens no seu conjunto, e em $5 \%(p=0,05)$, Quando se analisa isoladamente a realização de treinamentos para as precauções padrão. As instituições hospitalares se apresentaram, todavia, de forma diferente com significância estatística ao nível de $4 \%(p=0,04)$, para o conjunto de itens referentes a treinamento de pessoal para as precauções relativas à biossegurança, Quando separadas pela característica de se constituir ou não em campo de ensino. Também a realização de treinamentos não ocorre de forma 
distinta estatisticamente nos serviços hospitalares Quando levadas em conta as suas características como corpo clínico aberto ou fechado, clientela atendida (se SUS ou convênio privado e pagamento particular), predomínio da clientela, bem como sua localização geográfica.

No Que diz respeito ao item registros, observamos a ausência de mapa de risco em todas as instituições, presença de registro de casos de acidentes ocupacionais com materiais pérfuro-cortantes em sete $(28,0 \%)$, dos vinte e cinco hospitais e registro de cobertura da vacinação para hepatite B ausente em 20 (80,0\%) dos estabelecimentos. Esses mesmos registros, além de se constituírem em práticas pouco frequentes nos hospitais estudados ainda ocorrem de forma não diferenciada nos diversos grupos de hospitais. Exceções feitas às instituições agrupadas em com e sem grupo executivo de controle de $\mathrm{IH}$, as Quais se apresentam com diferenças estatisticamente significantes ao nível de 4,0\% $(p=0,04)$, detendo melhor resultado o primeiro grupo.

Também a realização/exigência de exame médico admissional é rotina pouco frequente nas instituições: em sete $(28,0 \%)$ dos hospitais esta prática era existente, ao passo Que a imunização dos profissionais para hepatite B era rotina em onze $(44,0 \%)$ dos serviços. Exames de PPD de profissionais atuantes no hospital não era realizado em nenhuma das instituições. $\mathrm{Na}$ análise individual de cada uma das Questões supramencionadas, detectamos, em contraponto, diferenças estatisticamente significantes no nível de $7,0 \%(p=0,07)$ para o subitem registro de acidentes com materiais pérfuro-cortantes, Quando os hospitais são caracterizados por tamanho em termos de número de leitos. Melhores resultados são apresentados pelos hospitais grandes, seguidos dos de médio e pequeno porte. Também apresentam diferenças estatisticamente significativas (aQui ao nível de 5,0\%- $p=0,05$ ) para o subitem, os hospitais classificados segundo campo de ensino, com melhores resultados para os Que se prestam às referidas atividades. O mesmo ocorre Quando os hospitais são subdivididos em com e sem grupo executor de I H ( $1,5 \%$ de significância - $p=0,015)$. Observa-se, adicionalmente, haver diferenças estatisticamente significantes no nível de 14,0\% $(p=0,14)$ Quando os hospitais são agrupados segundo a existência de $\mathrm{CCIH}$.

Os hospitais apresentam-se com diferenças significativas no nível de 3,0\% $(p=0,03)$ para as Questões agrupadas, Quando são categorizados em constituir-se ou não em campo de ensino e com e sem grupo executor de controle de infecção hospitalar, com melhores resultados para os primeiros respectivamente. E, ainda, no mesmo nível de significância, as instituições classificadas segundo tipo de atendimento geral ou especializadas, com melhores performances para os hospitais especializados.

Apesar de não se detectar diferenças estatisticamente significativas, pudemos verificar também para a referência à rotina de imunização para a hepatite $B$, frequência maior do Quesito, nos hospitais privados e filantrópicos a despeito das próprias entidades públicas. Mostraram-se, por outro lado, para o mesmo item, com resultados mais promissores e com diferenças estatísticas no nível de $3,0 \%(p=0,03)$, os hospitais campo de ensino. Também as instituições classificadas em gerais e especializadas, apresentaramse com diferenças estatisticamente significativas, mas ao nível de $7,0 \%(p=0,07)$. Nos hospitais especializados estavam mais presentes rotinas de vacinação para hepatite B do Quadro funcional,
Quando comparados com os hospitais gerais. Verificam-se, de igual modo, melhores resultados, com diferenças significativas ao mesmo nível de 7,0\% de significância, para o aspecto relativo à existência de rotinas de imunização, Quando as instituições dispunham de grupo executor de controle de IH.

Corpo clínico aberto ou fechado, tipo de clientela atendida, presença de $\mathrm{CCIH}$, localização geográfica das instituições, não distinguem os hospitais para as variáveis ora analisadas, tanto no Que concerne ao seu conjunto, Quanto para cada item tomado individualmente.

Com referência ao tópico em Que foram abordadas as Questões da instituição de isolamento para pacientes suspeitos de tuberculose e a existência de infra-estrutura para isolamentos para doenças transmissíveis, detectamos Que o primeiro item (prática de isolar pacientes suspeitos de tuberculose) foi referido em dezenove instituições (76,0\% dos hospitais), sem distinções estatisticamente significativas entre os diversos agrupamentos de serviços hospitalares utilizados para fins do estudo. Por fim, nenhum hospital dispunha de acomodações para isolamento de pacientes com doenças transmissíveis.

\section{DISCUSSÃO}

Sobre a infra-estrutura de biossegurança nos hospitais, iniciase com a discussão acerca da situação regional das Comissões de Controle de Infecção Hospitalar (CCIHs), e das Comissões Internas de Prevenção de Acidentes (CIPAs), posta a indubitável importância desses órgãos na conformação de uma adequada estrutura de biossegurança nos hospitais.

Assim nos chama a atenção a modesta presença dos referidos órgãos nas instituições estudadas (76,0\% no caso das $\mathrm{CCIHs)}$, dado este inferior ao de outras localidades brasileiras ${ }^{(3,5)}$. Por outro lado, verificamos maior precariedade da presença dos órgãos nos hospitais públicos, instituições Que em tese deveriam servir como agências diferenciadas no Que diz respeito ao controle de infecções e também no referente à uma estruturação mínima de cuidados à saúde do trabalhador. Situação Que pode ser considerada como digna de nota é a de Que além de reduzido, o número de enfermeiros atuantes nas $\mathrm{CCIHs}$ estudadas Que tiveram acesso a atualizações relativas ao tema, é ainda constituído por uma maioria de profissionais atuantes em hospitais privados e filantrópicos, contrariamente aos próprios hospitais públicos.

Se, de um lado, a situação dos hospitais da região em análise, no Que se refere à organização das $\mathrm{CCIHs}$, não se mostrou das mais promissoras, não é menos verdade Que as CIPAs, praticamente inexistentes (implementadas em apenas três - 12,0\% das instituições), também não desfrutem de melhor situação. ConQuanto tenhamos nos limitado à Questão da existência de uma noção mínima acerca de biossegurança por parte de pelo menos um de seus membros, os resultados mostraram-se pífios. Assim, os dados conjuntos das CCIHs e das poucas CIPAs, as Quais poderiam ter um papel coadjuvante de importância para a implementação e cobrança de uma estrutura apropriada de proteção contra os riscos biológicos, demonstram fragilidades Que merecem atenção por parte dos formuladores de políticas intra e extra-institucionais.

No tocante à presença de normas escritas, verificamos resultados pouco adequados. Uma possibilidade de explicação para a Quase 
ausência de normalizações sobre as precauções padrão talvez possa ser dada em parte por constituírem-se boa parte dos hospitais visitados, de pequeno porte $(56,0 \%)$. Esses serviços, de acordo com a literatura, apresentam maiores dificuldades em receber as normas dos órgãos de direito e adaptá-las às suas respectivas realidades $^{(6)}$. Dado importante, por outro lado, é aquele que mostra Que os hospitais públicos, mais diretamente ligados aos órgãos formuladores de políticas de prevenção e controle de infecções hospitalares e também de medidas de biossegurança, apresentam também graves debilidades no Que se refere à presença de normalizações.

Mais alentadora é a constatação de Que os hospitais Que se prestam a atividades de ensino apresentem-se com resultados melhores e com diferenças estatisticamente significativas dos seus congêneres Que não se prestam à aludida atividade. De igual modo, percebemos diferenciação nos hospitais segundo a existência de grupo executor de controle de IH o Que demonstra Que esses órgãos colaboram para uma melhor sistematização das normas afetas à biossegurança, pelo menos na nossa realidade e em respeito ao item aQui abordado.

No Que tange à presença de normatização por escrito acerca das precauções baseadas na transmissão podemos afirmar, contudo, Que o Quadro é ainda mais precário nas instituições estudadas, independentemente de sua classificação. Dados de hospitais paulistas mostram $43,0 \%$ deles com presença das referidas normas em contraponto aos resultados aqui detectados de $8,0 \%$, o Que demonstra importante variação regional ${ }^{(3)}$.

No tocante às normalizações escritas relativas ao atendimento de casos de acidentes de profissionais do hospital com materiais pérfuro-cortantes, por outro lado, percebemos Que um número pouco mais expressivo de hospitais as tinham $(32,0 \%)$, se comparados com as relativas às precauções padrão e baseadas na transmissão, mas ainda assim contingente Que pode ser considerado distanciado do ideal. Esse dado merece atenção levando-se em conta primeiro Que essas normas constituem-se, como de se esperar, em reproduções daquelas publicadas pelo Ministério da Saúde e, segundo, Que elas se encontram mais nos hospitais filantrópicos $(83,3 \%)$ do Que, mais uma vez, nos próprios hospitais públicos. Explicação plausível para a praticamente inexistência de normatizações relativas a condutas frente a acidentes com pérfurocortantes nos hospitais governamentais, talvez seja o fato de Que esse agrupamento de hospitais compreenda, coincidentemente, em sua maioria, na região, conforme já citado, os pequenos serviços para onde se encaminham os clientes de menor complexidade, o Que pode fazer com Que as preocupações com acidentes de profissionais com objetos pérfuro-cortantes sejam menores. Não devemos perder de vista, entretanto, Que o único hospital público de grande porte e responsável pelo atendimento de pacientes com demandas mais complexas se comparados aos dos pequenos nosocômios, não dispunha das referidas normalizações. Desta forma, no Que tange aos hospitais públicos em geral não podemos deixar de registrar Que parece ocorrer alguma lacuna na divulgação das instruções normativas por parte dos responsáveis técnicos pela área em nível governamental, posto Que teoricamente todas as instituições devem não apenas tê-las disponíveis, mas também aplicá-las Quando indicado e serem supervisionados pelo poder público Quanto à sua aplicação.Não há como negar, todavia, Que os resultados encontrados para o item treinamento parecem distanciados do Que se poderia considerar como alvissareiros. Apenas dez (40,0\%) das instituições estudadas referiram a realização de algum procedimento de educação em serviço relativo às precauções universais/padrão e nove $(36,0 \%)$ para as precauções baseadas na transmissão. Esses mesmos procedimentos, em sua maioria, dirigidos a enfermeiros e ocupacionais de enfermagem, deixando a descoberto outras categorias profissionais, dados coincidentes com estudo nacional levado a efeito há mais de dez anos atrás ${ }^{(7)}$. Treinamentos com vistas à triagem precoce de pacientes suspeitos de tuberculose, por outro lado, não foi objeto de educação continuada em nenhum dos hospitais visitados. A mesma modéstia é verificada Quanto ao número de hospitais Que se ocupam de registrar as ocorrências de acidentes de profissionais com materiais pérfuro-cortantes $(28,0 \%)$, o Que corrobora com a subnotificação dos casos, subnotificação esta Que nos hospitais pequenos e médios pode ocorrer pela errônea crença de Que, nessas instituições, o fato de os pacientes serem supostamente de menor complexidade, constituem-se em menores riscos para a veiculação de agentes infecciosos de importância ocupacional. De igual modo, também é frágil o registro de cobertura vacinal para hepatite B dos trabalhadores atuantes nas instituições. Explicação possível para a baixa preocupação nos hospitais com o registro de cobertura vacinal talvez relacione-se com a dinâmica de vacinação dos profissionais da saúde no Brasil. Observamos Que, em nosso País, o Ministério da Saúde disponibiliza, gratuitamente, as vacinas para a hepatite B e o gerenciamento dos referidos imunobiológicos fica a cargo das instâncias das secretarias municipais de saúde, as Quais são demandadas, para a vacinação dos profissionais, pelas respectivas instituições de saúde. Cumpre às secretarias registrarem o Quantitativo de doses utilizadas para proceder à reposição de seu estoque. Não cabe a essas últimas, contudo, o controle da situação vacinal dos empregados de cada hospital. Isto posto, Quando analisamos conjuntamente as informações do modesto número de hospitais Que registram a cobertura vacinal interrogamos se de fato os trabalhadores vem sendo adequadamente imunizados. Depreende-se, grosso modo, Que a Questão não se circunscreve, no caso específico dos hospitais aqui estudados, tanto ao acesso dos profissionais à vacina propriamente, posto Que conforme já citado, esta é oferecida de forma gratuita pelo poder público, mas numa assistematização de oferta e acompanhamento, pelo menos no Que concerne às instituições enQuanto empregadoras, o Que pode remeter à baixa cobertura em áreas de risco, desorganização e custos adicionais ao Estado.

Referente à existência de rotina de imunização dos profissionais contra a hepatite B verificamos Que somente nove $(36,0 \%)$ das instituições referem tê-la e diferenças significativas são detectadas Quando os hospitais são classificados em gerais e especializados, com melhores resultados para os últimos. Neste caso específico pudemos observar que os hospitais especializados aqui estudados eram todos voltados para a atenção à mulher, em particular no seu ciclo gravídico puerperal e à criança. Nossa hipótese é a de Que tal contexto faça com Que os profissionais atuantes nessas instituições sejam naturalmente mais afeitos ao tema vacina, pelo menos as concernentes às imunizações dos próprios usuários. Supomos Que, pela especificidade da clientela, a lida cotidiana com o assunto por parte dos profissionais influencie na preocupação também 
relacionada à vacinação dos próprios trabalhadores.

Ainda sobre a temática dos registros não foi encontrado em nenhuma das instituições visitadas mapa de riscos ambientais para a prevenção de acidentes de trabalho, mesmo nos hospitais de grande porte e naqueles que dispõem de CIPA. Malgrado os resultados surpreendam pela constatação de suposta despreocupação nos hospitais de sinalizar, através de recursos gráficos, os locais, situações e fatores de risco aos agentes biológicos, nos parece compreensível, posto Que a elaboração desses mapas é assunto relativamente recente nas instituições de saúde.

No Que concerne, por outro lado, à realização de exames médicos dos trabalhadores engajados nas atividades hospitalares, embora obrigatória por lei ${ }^{(8)}$, não se constitui em regra na maioria das instituições visitadas.

Referente à realização de PPD periódico em profissionais não reatores envolvidos na prática assistencial em áreas consideradas de risco para a tuberculose, observamos Que a medida não se constituía em rotina em nenhum dos hospitais pesquisados, dado esse Que ilustra Que a problemática da tuberculose ocupacional não parece fazer parte das preocupações nos serviços visitados. Embora não tenhamos elementos Que permitam comparar a realização dos aludidos exames em instituições nacionais com vistas a uma programação de prevenção de tuberculose ocupacional, cumpre-nos lembrar Que a doença é prevalente em nosso meio e vem aumentando sua incidência relacionada ao crescimento do número de casos de concomitância com o HIV e às dificuldades clínicas, propedêuticas e terapêuticas.

Verificamos, adicionalmente, Que nenhuma das instituições dispunha de infra-estrutura física específica para a instalação de isolamento de pacientes. Quadro Que demonstra frágil situação dos serviços hospitalares relativa ao atendimento de pacientes com doenças transmissíveis.

\section{REFERÊNCIAS}

I. Teixeira P, Valle S. Biossegurança - uma abordagem multidisciplinar. Rio de Janeiro: Fiocruz; 1998.

2. Secretaria de Saúde do Estado da Bahia (BA). Superintendência de Regulação, Atenção e Promoção de Saúde/Diretoria de Assistência a Saúde/ Coordenação de Gestão da Qualidade e Avaliação Tecnológica: Padrões de Qualidade da assistência para auto-avaliação hospitalar com foco no controle de Infecção Hospitalar - Portaria 1083. Salvador: Secretaria de Saúde do Estado da Bahia; 2001.

3. Oliveira TC. Controle de infecção hospitalar como indicador da Qualidade dos hospitais do município de Campinas e região [tese]. Campinas: Unicamp; 1997.

4. SAS INSTITUTE INC. SAS/STAT User's Guide. Release 6.03.
Cary: SAS Institute Inc; 1995.

5. Gontijo Júnior OM. Avaliação das comissões de controle de Infecção Hospitalar em Belo Horizonte. Proposta para Incremento da Resolutividade [tese]. Belo Horizonte: Faculdade de Medicina, Universidade Federal de Minas Gerais; 1991.

6. Celentano DD. Diffusion and adoption of CDC guidelines for the prevention and control of nosocomial infections in US hospitals. Infection Control 1987; 8(10): 415-23.

7. Pereira MS, Moriya TM. Infecção hospitalar. Estrutura básica e controle. 2a ed. Goiânia: AB; 1995.

8. Ministério do Trabalho e Emprego (BR). Portaria $n^{\circ} 24$, de $29 /$ 12/94. Brasília: Ministério do Trabalho e Emprego; 1994. 


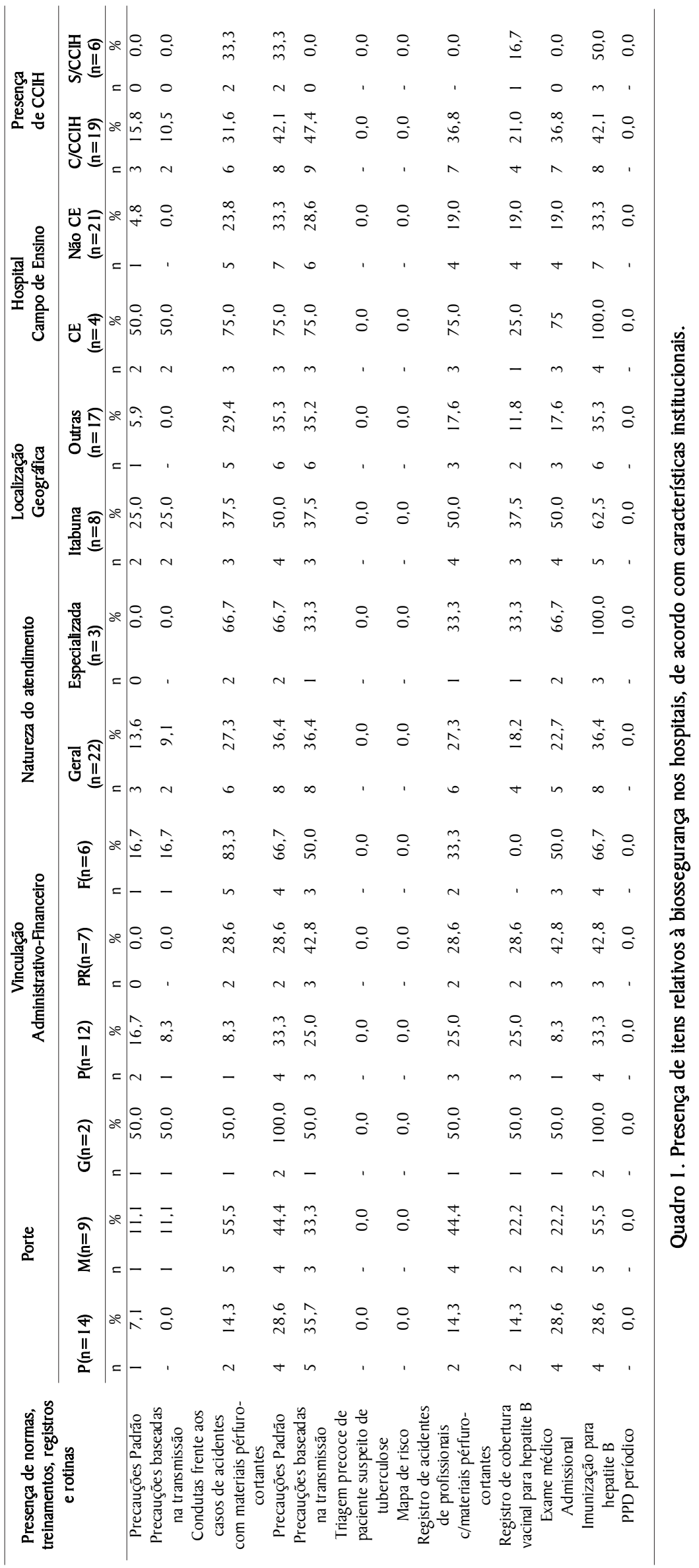

